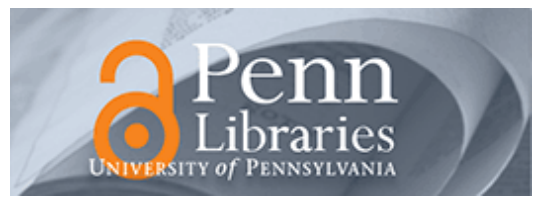

University of Pennsylvania ScholarlyCommons

\title{
\#GirlsLikeUs: Trans advocacy and community building online
}

Sarah Jackson

Follow this and additional works at: https://repository.upenn.edu/asc_papers

Part of the Communication Commons

\section{Recommended Citation}

Jackson, S. (2018). \#GirlsLikeUs: Trans advocacy and community building online. New Media and Society, 20 (5), 1868-1888. https://doi.org/10.1177/1461444817709276

At the time of publication, author Sarah Janet Jackson] was affiliated with Northeastern University. Currently, She is a faculty member at the Annenberg School for Communication at the University of Pennsylvania.

This paper is posted at ScholarlyCommons. https://repository.upenn.edu/asc_papers/774

For more information, please contact repository@pobox.upenn.edu. 


\title{
\#GirlsLikeUs: Trans advocacy and community building online
}

\begin{abstract}
In this research we examine the advocacy and community building of transgender women on Twitter through methods of network and discourse analysis and the theory of networked counterpublics. By highlighting the network structure and discursive meaning-making of the \#GirlsLikeUs network, we argue that the digital labor of trans women, and especially trans women of color, represents the vanguard of struggles over self-definition. We find that trans women on Twitter, led by Janet Mock and Laverne Cox, and in response to histories of misrepresentation and ongoing marginalization and violence, deliberately curate an intersectional networked counterpublic that works to legitimize and support trans identities and advocate for trans autonomy in larger publics and counterpublics.
\end{abstract}

\section{Keywords}

transgender, counterpublics, Twitter, hashtags, online advocacy, network analysis, discourse analysis, \#GirlsLikeUs

Disciplines

Communication | Social and Behavioral Sciences

\section{Comments}

At the time of publication, author Sarah Janet Jackson] was affiliated with Northeastern University. Currently, She is a faculty member at the Annenberg School for Communication at the University of Pennsylvania. 


\begin{abstract}
In this research we examine the advocacy and community building of transgender women on Twitter through methods of network and discourse analysis and the theory of networked counterpublics. By highlighting the network structure and discursive meaning-making of the \#GirlsLikeUs network, we argue that the digital labor of trans women, and especially trans women of color, represents the vanguard of struggles over self-definition. We find that trans women on Twitter, led by Janet Mock and Laverne Cox, and in response to histories of misrepresentation and ongoing marginalization and violence, deliberately curate an intersectional networked counterpublic that works to legitimize and support trans identities and advocate for trans autonomy in larger publics and counterpublics.
\end{abstract}

Keywords: transgender, counterpublics, Twitter, hashtags, online advocacy, network analysis, discourse analysis, \#GirlsLikeUs

\title{
Introduction
}

In 2012, following several high profile murders and suicides of queer and trans youth, Janet Mock was moved to become a more outspoken trans activist. ${ }^{1}$ As former web editor for fashion magazine Marie Claire, Mock used her cultural capital and digital tools like YouTube videos and hashtags, to reach out to other trans women with messages of support. In this article we use network and discourse analysis to illustrate how trans women's support and advocacy online epitomizes the complications and possibilities of networked counterpublics. We apply the concept of network counterpublics as defined in the work of Jackson and Foucault Welles (2015) who extend the concepts of the networked public sphere and counterpublics to the cultural work and interventions of historically marginalized citizens on Twitter (Asen and Brouwer, 2001; Benkler, 2006).

While Mock has since become something of a celebrity representative for trans women in the U.S., her digital engagement and visibility were not always so notable. When Mock initially used the hashtag \#GirlsLikeUs she had just under 3,000 followers - a modest following surpassed by many academics, journalists, and ordinary users. In explaining the origin of the 
hashtag, Mock describes her support of Jenna Talackova, a contestant disqualified from the Miss Universe Pageant for, in the words of the pageant officials, "not being a natural born female." Mock's desire to help Talackova define herself in a mainstream space led to the creation of the hashtag and what would soon become a network of trans feminist community building and advocacy. ${ }^{2}$ On May 28, 2012, Mock explained on her personal blog:

So I shared Jenna's petition on Twitter, and said: Please sign \& share this women's rights petition in support of transgender beauty queen Jenna Talackova \& \#girlslikeus: ow.1y/9TYc6b 27 Mar 12 And that was the online birth of \#girlslikeus. I didn't think it over, it wasn't a major push, but \#girlslikeus felt right. Remarkably a few more women - some well-known, others not - shared the petition and began sharing their stories of being deemed un-real, being called out, working it, fighting for what's right, wanting to transition, dreaming to do this, accomplishing that.... \#girlslikeus soon grew beyond me... my dream came true: \#girlslikeus was used on its own without my @janetmock handle in it. It had a life of its own. (Mock, 2012)

Mock has also credited CeCe McDonald, a formerly imprisoned trans woman from Minnesota who we discuss in greater detail later in this article, as an inspiration for the hashtag, stating on Twitter, “\#BecauseofCeCe I was inspired to begin using the phrasing \#GirlsLikeUs which led to a social media visibility movement.\#CeCeIsFree," (Mock, 2014a). As indicated in these narratives, Mock created the hashtag spontaneously in an effort to support the rights of other trans women - from beauty pageants to court rooms - and was happy to see the hashtag evolve beyond her (though as we detail below, she remained central to \#GirlsLikeUs network through the choices of members within it).

Others indeed quickly embraced the hashtag, using it to discuss everything from the desire to transition, to the violence of being outed without consent, to professional goals. In the creation and evolution of \#GirlsLikeUs we see what Michael Warner (2002) has called the "world-making" power of publics, that is the discursive self-organization of a public among 
strangers that reflexively speaks to the historical and contemporary contexts of its makers and observers. Through the discursive contributions of a self-organized community of trans women, the hashtag became a space for counterpublic engagement; together users created a network in which conversations that nurture in-group political and cultural projects can and do reach beyond it.

Other work on counterpublics has illustrated that members of society attempting to create sweeping political change can successfully levy the technological affordances of Twitter to challenge dominant discourses of power and draw attention to issues and incidences that affect oppressed communities (Jackson and Foucault Welles, 2016; Loader and Mercea, 2011; Papacharissi and de Fatima Oliveira, 2012). This work has primarily focused on cases of networked counterpublic activism that very quickly received mainstream results. By detailing the evolution of the \#GirlsLikeUs counterpublic outside mainstream attention and primarily for purposes of in-group solidarity, we provide important nuance to the existing scholarship.

As Squires (2002) has detailed, counterpublics "emerge not only in reaction to oppression from the state or dominant public spheres, but also in relation to the internal politics of that particular [counter]public sphere and its material and cultural resources." As a result, some members of counterpublics occupy more or less subaltern positions than others. In the case of trans women who are members of larger LGBTQ and feminist counterpublics, and trans women of color who also occupy important roles in race and ethnicity-based counterpublics, their intersectional identities result in simultaneous marginalization in all three communities (Crenshaw, 1991; Johnson, 2013).

Given historical and contemporary exclusions, both in the mainstream and within identity-based counterpublics, we argue that the communication constructed by and for trans 
women online illustrates some of the most subaltern. We offer this study of trans women's networked labor both in the spirit of recognizing the layered ways counterpublics function, and as a response to activists and scholars who have called for a centering of the " $\mathrm{T}$ " in LGBT in communicative analysis (Capuzza and Spencer, 2015).

\section{Trans Women, Media, and Representation}

In 2015, trans activist Jennicet Gutiérrez was booed by a largely cisgender crowd and physically removed from an LGBTQ White House event after speaking out about the disproportionate violence faced by trans women in United States immigration detention centers. Gutiérrez's words and interruption of President Barack Obama were met with harsh criticism from many organizations that bill themselves as central to LGBTQ equality efforts. Gutiérrez was chastised for a "lack of civility" and "rudeness" by the LGBTQ magazine the Advocate, which compared her asking Obama to address the torture and rape of transgender immigrants in detention centers to Kanye West's infamous interruption of Taylor Swift at the 2009 MTV Video Music Awards (Ennis, 2015). For trans women, and especially trans women of color, the moment was indicative of a painful pattern of derision by members of the assimilationist LGBTQ counterpublic who view trans critique as disruptive. ${ }^{3}$

Beyond explicitly political and activist spaces, the representation of trans women as disruptive - even dangerous - to civil society is evident in how trans women are portrayed on the U.S.'s big and small screens. Representation of trans women, and trans women of color in particular, in news and popular culture has long been one of stereotyped hypersexual tricksters whose victimization at the hands of cisgender men is framed as a natural or deserved consequence of their disruptive identities (Schilt and Westbrook, 2009; Serano, 2007). In the early 1990s, trans women became regular objects of fascination on daytime talk shows. The 
Jerry Springer Show, for example, was frequent fodder for scholars interested in trans representation - representations that tied trans identities to fear, deception, and freakishness (Gamson, 1998). These media representations have both influenced and been influenced by larger cultural and political narratives about trans identity. In U.S. courts trans women have been deemed deviant and held responsible for the violence and discrimination they face. In the late 1990s, for example, the "gay and trans panic" defense became popular among some attorneys seeking to justify their clients' violent crimes against members of the queer and trans community (Lee and Kwan, 2014).

In the last thirty years, the visibility of transgender Americans has shifted significantly. In 2007, trans advocates were successful in including language addressing gender identity as a possible site of employment discrimination as the Employee Non-Discrimination Act (ENDA) was debated in Congress. Yet this language was subsequently removed because lesbian and gay special interest groups did not think the bill could pass with its inclusion (Vitulli, 2010). The bill still failed. It was not until 2013 that a new ENDA including trans identity was signed by President Barack Obama. Also in 2013, the Netflix original series Orange is the New Black became a runaway hit, in part because of Laverne Cox's compassionate portrayal of an incarcerated Black trans woman. The same year, in a statement widely circulated online, military whistleblower Chelsea Manning came out as a trans woman. In 2014, California became the first (and only) state to pass a law banning the gay and trans panic defense (Martohardjono and Young, 2016). The very public 2015 transition of Olympic Gold medalist Caitlyn Jenner reached a crescendo when Jenner tweeted an image of her now famous "Call me Caitlyn" Vanity Fair cover, helping further transgender visibility (Statista, 2015). 
Despite such notable visibility and Time magazine's cover proclamation in 2014 that the United States had reached a "Transgender Tipping Point," Capuzza (2015) notes that a "quantitative increase in media attention did not equate with challenges to cisnormativity.” In fact, trans women continue to negotiate unique threats to life and health, which are further multiplied at the intersections of race, class, and sexuality. Alongside the advances and visibility of the last five years, trans women continue to be murdered at a startling rate. According to a 2015 report from the Southern Poverty Law Center, trans women are the group most victimized by violent hate crimes in the United States (Southern Poverty Law Center, 2015). During the first two months of 2015, transgender women of color were murdered at a rate of almost one per week in the United States (Michaels, 2015). Further, these statistics do not include the widespread harassment, abuse, and harm that trans women survive as they move about the word. In today's popular media, Hollywood is notorious for casting cisgender, heterosexual actors as trans women. Jared Leto in Dallas Buyers Club (2013), Felicity Huffman in Transamerica (2005), Eddie Redmayne in The Danish Girl (2015), and Jeffrey Tambour in Transparent (2014), have all drawn critique from trans and allied communities for how such casting continues to marginalize trans actors. ${ }^{4}$ Likewise, the increasing visibility of trans women did not prevent the makers of the 2015 historical drama Stonewall, about the uprising that birthed the LGBTQ rights movement, from erasing the role of trans women of color in the activism of 1960s Greenwich Village (Mendelson, 2015). Much-publicized critiques of the film's whitewashing of the Stonewall Rebellion (using the hashtag \#NotMyStonewall) led to a boycott of the film and its subsequent failure at the box office. Many boycotters instead chose to fund Happy Birthday Marsha!, an independent film about Marsha P. Johnson, a Black trans woman 
who was a central figure in the Stonewall riot and queer and trans activist circles in New York City (Shorey, 2015).

As this example illustrates, visibility and debate on trans issues, and the simultaneous frequency of anti-trans acts of violence and erasure, have prompted increased advocacy by trans women through online media. This advocacy has continued in the wake of the 2016 election and the anti-trans discourse and policies threatened by the Trump administration. As we demonstrate herein, online community building and advocacy among trans social media users and activists has been important in extending and nurturing a trans counterpublic that provides life-affirming support to trans women online and that actively engages U.S. culture and national politics.

While still too few, a growing body of research has considered the important role of online trans advocacy in challenging and reshaping the way media makers, and the nation, communicate about trans identities. In research that focuses on Black trans and queer women's use of digital media, Bailey (2016) has argued that independently-produced media help gender marginalized people to "survive and thrive" in an otherwise hostile biomedical landscape. In particular, she argues that the digital media production of trans black women engender expanded health praxis that aid in the physical survival of queer and trans bodies. Calvacante (2016) has similarly found that transgender-centered websites enable "architectures of organized care and concern" as trans counterpublics readily support one another through physical transition in an interactive media environment made possible through new media outlets. Rawson (2014) compellingly argues that transgender worldmaking online is an especially important site of historical activism because, “cyberspace provides a revolutionary tool for creating, sharing, and preserving trans histories that would otherwise remain untold.” Barnett (2015) has detailed how blogs authored by trans people have created a "transsexual counterpublic" in which the human 
body is constructed as a natural and continual site of transformation in opposition to dominant constructions of the body and gender as normatively immutable.

We too view the work of trans advocates online as a particularly fruitful space for the study of counterpublic identity politics because on social media. Unlike in news and entertainment media spaces, trans women can communicate about and construct their identities and experiences without the fraught, incomplete, and transphobic mediation of mainstream narratives (Booth, 2015). Further, we suggest that the digital discourse of \#GirlsLikeUs extends the characteristics of transactivist literature described by Hundley and Rodriguez (2009) as various members of the \#GirlsLikeUs network share their stories, offer each other support, and advocate for trans histories, worlds, and rights. These characteristics include organizing a community through identity construction, the celebration of diverse trans voices, and a polysemy through which members of the network create multivalent appeals to one another and to the larger public. Through an examination of the \#GirlsLikeUs network and related hashtags, we illustrate how for trans women Twitter is an important site of countercultural practice, intervention, and representation.

\section{Approaches and Methods}

We approach this work using the interdisciplinary frameworks of network science, cultural studies, and digital humanities and a commitment to mixed methods examinations of new media phenomena. The theories and analytic methods of network science allow us to capture and parse the large and ever-growing corpus of evidence of trans women's advocacy online. Transforming online discourse into networks of individuals linked by communication 
acts makes it possible to understand the interconnected nature of online conversations, while also whittling down an overwhelming volume of data to a manageable size.

On Twitter alone, the hashtag \#GirlsLikeUs has appeared in almost 150,000 tweets. Even at just 140 characters per tweet, it is intractable to analyze this volume of data. Instead, we captured a 10\% random ("garden hose") sample of tweets sent between July 2013 and June 2015 that contained the hashtag \#GirlsLikeUs. We transformed these 14,991 tweets into a network of users connected by retweets and mentions, limiting the sample to only those users retweeted or mentioned at least one time (removing isolates). The resulting network included 6,857 nodes (Twitter users) connected by 10,253 links (retweets and mentions). From these, we extracted tweets generated by or about the top 10 most retweeted or mentioned nodes (highest in-degree) for in-depth discourse analysis. This sampling technique has been used elsewhere to study Twitter activism networks and, because of uneven degree distributions in social networks, ensures that our focal sample included tweets that were disproportionately more likely to be seen within the network as a whole (Barabási and Albert, 1999; Jackson and Foucault Welles, 2015, 2016; Romero and Kleinberg, 2010). Of note, this sampling technique is agnostic to the content of the tweets (other than including \#GirlsLikeUs) or identities of the individuals that compose them, allowing us to select individuals and cases for analysis without any a priori notions about who ought to be included.

After sampling \#GirlsLikeUs tweets for analysis, we used emergent discourse analysis to fully explore the significance of this data. It is in the bridging of quantitative data with humanities approaches that the unique story of the power of these tweets can be told. Discursive analysis of counterpublic meaning-making allows us to explore the significance of hashtags beyond quantified popularity and digital curation to highlight the social and political labor 
undertaken by members of the \#GirlsLikeUs network. This approach is a constructivist one which recognizes that discourse constructs reality by making ideas and events meaningful in particular ways that uphold, and/or challenge cultural ideologies.

Thus, our discourse analysis of \#GirlsLikeUs and its co-occurring hashtags and narratives highlights the agency and power of trans women online who are helping to define gendered citizenship and cultural belonging. We examine discourse from the most popular and influential tweets and members of our network (as determined in our network analysis), for narratives of belonging and exclusion, interpersonal connections, storytelling, social and political observation, and contextualizing to understand how members of the counterpublic use the hashtag to frame particular issues and experiences in attempts to participate in the counterpublic and public spheres.

\section{Network Characteristics of \#GirlsLikeUs}

As a network of tweeters connected by retweets and mentions, the \#GirlsLikeUs network has several notable features. The conversation was disproportionately driven by Janet Mock and actress Laverne Cox, who were retweeted thousands of times more often than the next most retweeted people in the network. More than $15 \%$ of the re-tweets and mentions in the network were generated by or about Mock, and nearly $9 \%$ by or about Cox. Their centrality is reflective of their early and frequent use of \#GirlsLikeUs, as well as their growing celebrity during the time our data were collected. Given their centrality, \#GirlsLikeUs is a broadcast network, or a network in which most people retweet messages authored by a small number of prominent users (Smith et al., 2014). Thus, almost one quarter of the tweets we examined contained content originally authored by either Mock or Cox. Through retweeting that content, users actively centered and uplifted Mock and Cox, illustrating the organic nature of leadership in the network. 
Another quarter of tweets in the network originated from the next 8 most retweeted users, which include ordinary trans women, advocates and public figures like Carmen Carrara and Geena Rocero, trans activist groups like Trans March, and several media outlets that published articles about trans women during the time our data were collected. Although it is not uncommon to find extremely skewed degree distributions in social networks in general (Barabási and Albert, 1999) and Twitter networks in particular (Romero and Kleinberg, 2010), that this network was centered on a diversity of trans women's voices is reflective of the power of the medium as a platform for marginalized voices.

Though their tweets were disproportionately influential, higher profile users make up only a small fraction of the total population within the \#GirlsLikeUs network. In addition to Mock and Cox, whose celebrity grew along with the network, there were thousands of regular people who tweeted with the hashtag. On an individual basis, these users received relatively little attention, with an average of one or two retweets or mentions each. But, taken as a whole, their tweets accounted for almost half of the network activity. Thus, we find evidence to support Mock's above observation that the hashtag was used widely without her Twitter handle, even as the network as a whole positioned her centrally.

The network grew steadily, throughout the two years we observed, with an average of dozens to hundreds of new tweets appearing per day. There were two spikes in activity in response to exogenous events involving trans women - once in December 2014, when Mock, Cox and twelve other trans women appeared on the cover of trans-focused publication CANDY magazine, and June 2015, when Caitlyn Jenner appeared on the cover of Vanity Fair magazine.

Looking more broadly across the network, there is a remarkable level of cohesion among those using the \#GirlsLikeUs hashtag, with many people tweeting at or about one another, in 
addition to engaging with the most retweeted members of the network. The average distance between members of the network is just slightly over three, meaning that any given person in the network is in communication with any other through just two intermediaries. Combined with the disproportionate number of tweets originating from Mock and Cox, this suggests that most people in the network are either engaging directly with Mock and Cox, or engaging with people who are.

The network structure also provides evidence of a cohesive conversation, stemming from Mock and Cox, and centered, as we detail below, on inclusion and affirmation of trans women. Consider the following network visualization: Mock and Cox, and their followers constitute the majority (just over 75\%) of the network, visualized in purple, wrapping around the upper right portion of the graph. In the lower left, visualized in green, we see a sub-community that is almost entirely detached from the rest of the network. This sub-community is centered on two selfidentified conservative women, @xoCAMILLAxo and @Meeech_L, who tweet primarily about conservative political issues. They tweet frequently, and are often retweeted by users who are neither in conversation with the main portion of the \#GirlsLikeUs network, nor with one another. 
Figure 1. Visualization of \#GirlsLikeUs retweet and mention network. This graph was generated in Gephi, using the Fruchterman-Reingold force directed layout algorithm.

Of note, @xoCAMILLAxo and @ Meeeech_L turn up in our search results, not because they use the hashtag \#GirlsLikeUs in their tweets, but because both had set their profile locations to \#GirlsLikeUs. By including \#GirlsLikeUs in the location settings of their twitter profiles, these users add the hashtag as a form of metadata to all their content. This metadata of location, tweet identification numbers, and timestamp are included in search results regardless of whether 
or not the hashtag appears in the text of a tweet. By setting their location to \#GirlsLikeUs, these users became part of the hashtag network even if only on the periphery.

Because both women tweet frequently, and their tweets turn up in search results for the hashtag, we can be reasonably sure that the broader community of tweeters using \#GirlsLikeUs is aware of their existence. Thus, @xoCAMILLAxo and @Meeeech_L's near complete separation from the rest of the \#GirlsLikeUs network is no accident -- the community has assessed the content of their tweets and chosen not to engage with it. This divide underscores that \#GirlsLikeUs is predominantly a bounded community, centered on trans women with progressive and left-leaning politics. For the vast majority of Twitter users engaging in conversations with the hashtag, \#GirlsLikeUs is a space to talk through how trans identity, along with other forms of inequality including racism and poverty, impacts daily life. Conservative users who reject the importance of these issues are summarily ignored. ${ }^{5}$

While Mock and Cox remain central to the network and thus help shape the scope of the conversation therein, neither they nor other members of the network engage in any policing behavior or attempt to control the discourse of others who use the hashtag. In other words, conservative users of the hashtag are ignored, but they are not reprimanded, while other users are actively rewarded by one another through attention and connection.

\section{Narrating the World of \#GirlsLikeUs}

Through our discourse analysis of the most popular tweets and accounts in the network we discovered that trans women use \#GirlsLikeUs in three primary ways: 1) to connect with one another on every day, often mundane, experiences, 2) as a way to advocate for trans issues and rights (particularly through critiques of mainstream representations of trans people and anti-trans 
violence), and 3) to celebrate the accomplishments of trans women. This third use of the hashtag often overlaps with the previous two.

\section{Building community}

Members of the \#GirlsLikeUs network regularly tweet about their day to day lives and challenges. For example, trans businesswoman @angelicaross shared, "I'm regaining at least 20 hours of my life back a week moving to Chicago! More time for my spirit, tennis, yoga \& friends \#GirlsLikeUs" (Ross, 2013). This kind of positively-toned share is frequent in the network, as are words of support and encouragement. When trans teen Cassidy Lynn Campbell posted a tearful video about the online bullying she was experiencing after being named homecoming queen, she received an outpouring of support from members of the network, including Janet Mock who tweeted, "Dry those tears, babydoll. You are a bright, shining star, @xocassidylynn! \#girlslikeus," (Mock, 2013). This rapport building between members of the network, regardless of status, does important community-building work, shaping cultural solidarity and providing important emotional and psychological support (Bailey, 2015).

Further, discourse about quotidian experiences, which includes, for example, accounts of sick relatives and breakups, works to both center and normalize trans voices and experiences by illustrating how trans women's lives are not as unrelatable as popular culture would suggest. One user tweeted, "Sure fire way to cheer myself up: a glass of wine and streaming the @ moschino Fall Winter 14-15 show. \#girlslikeus \#fashion"; while another shared that, "This will be my 1st Easter alone b/c my anti-trans family who claim to love Jesus refuses to accept me. \#girlslikeus," and another queried, 'I still haven't watched TransParent. Is it worth watching? I've heard mixed reviews from \#girlslikeus."6 
These examples illustrate the diverse and ordinary ways trans women used the hashtag to document their daily lives. We see \#GirlsLikeUs relaxing, sharing familial difficulties, and asking for recommendations on entertainment. Further, as heinz (2015) has noted, feelings of isolation are one of the most frequent reoccurring mental health issues among trans people. In building a community that transcends distances through \#GirlsLikeUs, Twitter has become a space for trans women to both normalize their identities to observers and create healthy interpersonal connections by locating other trans people, finding social support, and sharing the "micro-stresses" of trans living.

\section{Creating change}

\#GirlsLikeUs tweets that focus on advocating for trans issues and rights do so by elevating the voices and history of trans women and sharing facts and information about trans experiences with injustice. These tweets are educational, a call to action, or both. For example, Trans March (@TransMarch) a San Francisco organization that works to "inspire all trans and gender non-conforming people to realize a world where we are safe, loved, and empowered," regularly tweets educational and biographical information about trans activists and figures like Martha P. Johnson, Jazzie Collins, Billie Cooper, and Chelsea Manning (San Francisco Trans March, n.d.). 


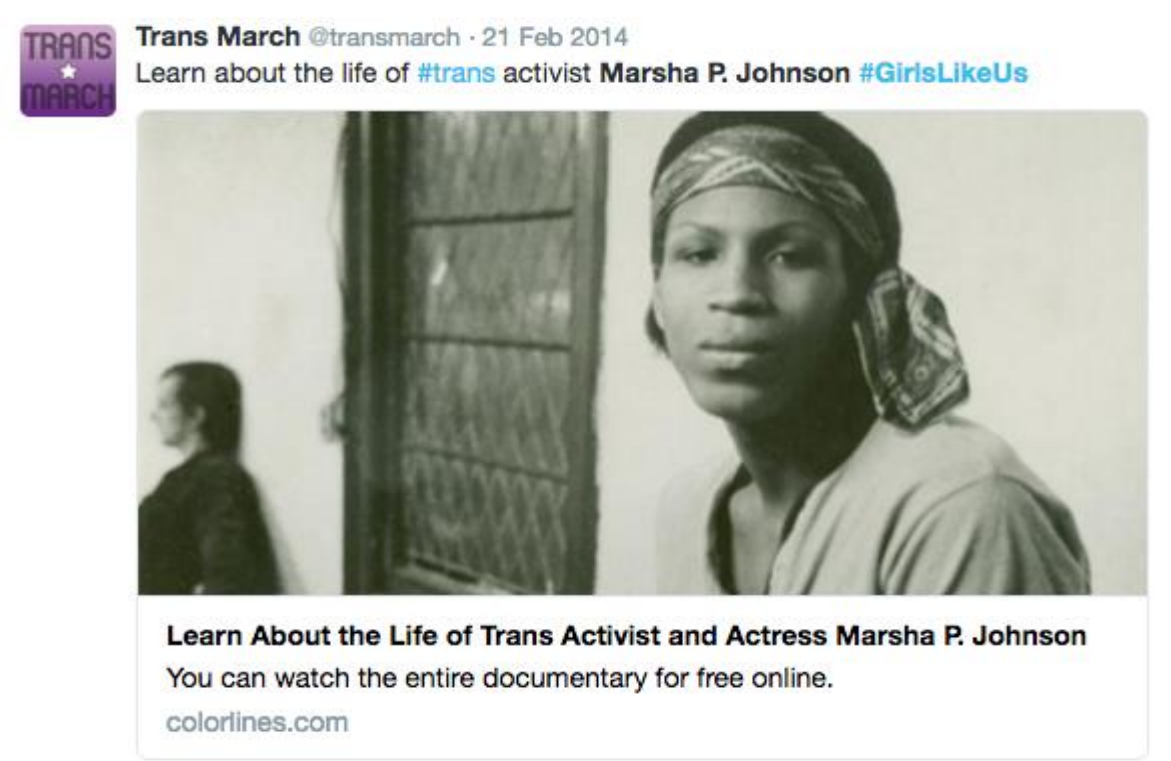

Figure 2: Trans March \#GirlsLikeUs tweet about activist Marsha P. Johnson

Tweets in this category also work to connect issues of trans liberation to intersectional concerns of poverty, racism, and sexism. For example, Janet Mock connected the case of CeCe McDonald to that of Trayvon Martin, the Black Florida teenager killed by neighborhood watchman George Zimmerman. By using the hashtag \#JusticeforTrayvon alongside \#GirlLikeUs and \#FreeCeCe, Mock discursively works to illustrate the connection between anti-Black violence and trans identities often left out of conversations on racial profiling. At the same time, Mock makes the issue of anti-Black violence, one too often excluded from the mainstream LGBT movement, central to her brand of trans advocacy.

Janet Mock @janetmock · 5 Aug 2013

NEW blog by @Free_CeCe on \#justicefortrayvon, racism \& her sense of "survivor's guilt" supportcece.wordpress.com/2013/08/04/inj... \#freecece \#girlslikeus \#twoc

Injury and Insult: Trayon Martin, racism in the syste... As I sit and watch Michelle Alexander and Chris Hayes have a conversation about race, as well as all of the nation in light of the George Zimmerman acquittal, it ... supportcece.wordpress.com 
Figure 3: Janet Mock tweets \#FreeCeCe, \#JusticeforTrayvon, and \#GirlsLikeUs

In a similarly intersectional spirit, Laverne Cox uses many of her public interviews about the hit Netflix series Orange is the New Black (OITNB) to discuss issues facing trans prisoners and to advocate for prison reform. Cox often uses the mantle of sharing content about OITNB to introduce and discuss these more serious topics. For example, after her first appearance on the Melissa Harris Perry Show, Cox tweeted, "Check out the video of my \#nerdland debut today. We talk about @OITNB and prison policy http://t.co/SuE1pJvuej@MHPshow \#girlslikeus,” (Cox, 2013). Cox connects the hashtag to a policy conversation on incarceration while maintaining the casual tone of the \#GirlsLikeUs network and calling in OITNB (@OITNB) and Melissa Harris Perry Show (\#Nerdland) fans. This practice illustrates how the hashtag is used as a prompt to a community of fans and allies who because of shared ideological and taste cultures are invested in the lives, successes, and inequalities faced by trans women.

What is notable in these examples, and throughout \#GirlsLikeUs advocacy, is the centering of intersectional experience. Women of color activists and stories about intersectional activism are widely visible in the \#GirlsLikeUs network. Not only does the discourse created in the network contribute to an educational project on trans rights and issues, it also works toward framing the solutions to anti-trans violence and transphobia as inextricably tied to fights against racism and sexism. Such hashtagged advocacy contributes to what Florini (2014) has called a "recontextualization" of mainstream social movement memory and in this case, recentering trans experiences in civil rights, LGBT, and feminist projects.

\section{Celebrating trans lives}

\#GirlsLikeUs users frequently engage in celebrating trans women's accomplishments and visibility. This practice overlaps with advocacy narratives that celebrate trans activists and 
community-building narratives that highlight the rise and accomplishments of trans women. For example, trans model Geena Rocero publicized a photoshoot in Glamour Magazine using \#GirlsLikeUs alongside the hashtag for her own trans advocacy organization Gender Proud, and the hashtag \#TransRevolution. Here, we see Rocero connecting her accomplishments as a trans women and public personality - a profile in a mainstream women's magazine - to trans advocacy work offline and to the digital community constructed through \#GirlsLikeUs. As is common in the \#GirlsLikeUs network, Rocero's tweet was responded to with words of encouragement, including from Janet Mock who replied, "gorgeous + glowing! Can't wait to pick up my copy! \#genderproud \#girlslikeus," (Mock, 2014b).

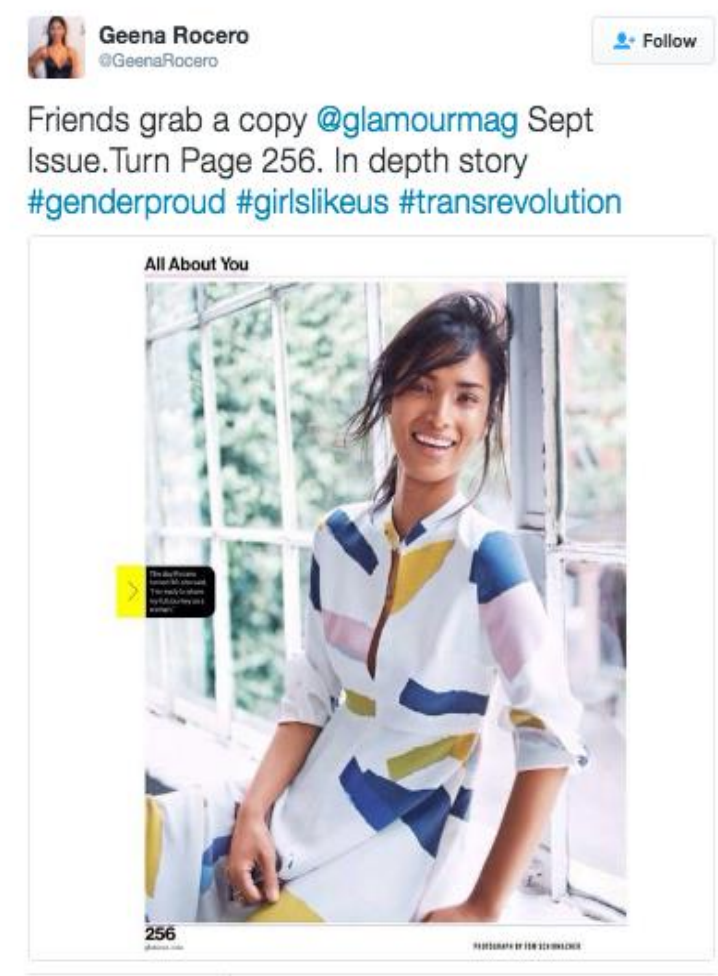

Figure 4: Geena Rocero tweets about her Glamour magazine profile with \#GirlsLikeUs \#GenderProud and \#TransRevolution 
Many members of the \#GirlsLikeUs network, including those with less visibility, use the hashtag to celebrate their accomplishments, including new jobs, a new step in gender confirmation, or a cross-country move. Here again, we see the overlap of how the hashtag works to build community from within while centering and normalizing trans lives to outside observers. For example, Angelica Ross tweeted that she was, “just interviewed by @Forbes on \#TransTechSocial \& my journey as a Black trans entrepreneur! Thanks @ClairJoyFarley! \#GirlsLikeUs," (Ross, 2014). This tweet, while a celebration of her own accomplishment (being interviewed by Forbes), also works to build community by mentioning Trans Tech Social, the development and employment community Ross founded for trans and gender nonconforming people. By thanking author Clair Farley, Ross signals her as an advocate for trans people in business and media.

\section{Co-occurring Hashtags and Broadening a Counterpublic}

As the above examples illustrate, one of our descriptive findings is that the \#GirlsLikeUs network makes frequent use of co-occurring hashtags to broaden conversations about trans women's experiences. Considering the connections between \#GirlsLikeUs and broader conversations about trans women, we find a number of other co-occurring hashtags that both reinforce and extend the conversation centered on \#GirlsLikeUs. The most common co-occurring hashtags include references to trans people, trans women, and trans women of color (e.g. \#trans, \#Tgirl, \#twoc), references to specific trans women in the news (e.g. \#CallMeCaitlyn referring to Caitlyn Jenner, and \#IslanNettles, a Black trans woman murdered in a 2013 hate crime), and references to general social issyesthat are important to members of the trans counterpublic (e.g. \#education, \#tolerance). 
Of particular interest are co-occurring hashtags popularized by network influencers Janet Mock and Laverne Cox. Like other notable advocates for change whose membership in counterpublics and experiences of marginalization shaped their engagement with mainstream politics and culture, Mock and Cox's growing celebrity gave them access to mainstream discourse despite the very real exclusions they and other members of their community face. As Jackson (2014) details in her examination of Black celebrity dissent and activism, celebrities who bridge subaltern and mainstream communities often become representatives for the publicization of counterpublic narratives and politics. In Mock and Cox's case, these women elevated the hashtags \#FreeCeCe, \#RedefiningRealness, and \#TransIsBeautiful alongside their \#GirlsLikeUs tweets.

\section{\#FreeCeCe}

In June 2012, 23-year-old CeCe McDonald was accosted with racist, transphobic, and homophobic slurs by bar patrons in downtown Minneapolis, Minnesota. An attacker threw a bottle at McDonald's head, which shattered and created a large gash on her face. McDonald used a pair of scissors to defend herself and one of her attackers, a member of a white supremacist gang, was killed. McDonald was sentenced to three and a half years in a men's prison. Her trial and subsequent imprisonment raised many questions about the differential treatment Black trans women experience in the U.S. legal system - particularly as related to self-defense and concepts of victimhood (Hill, 2012). Members of the trans feminist counterpublic we examine here used social media to share McDonald's story and protest her imprisonment, identifying her as a Girl Like Us.

In considering the discursive power of \#FreeCeCe and its variations (e.g. \#Free_CeCe, \#CeCeisFree, \#BecauseofCeCe), we see the connection of the community building and advocacy 
work characteristic of the \#GirlsLikeUs network extended to a specific criminal justice case. In this case, the two hashtags work to center women like CeCe McDonald - Black, femme, young, and incarcerated - in the trans counterpublic, and to make a specific demand of a legal system that is anything but fair in its treatment of Black trans women (Crenshaw, 1991; End Solitary campaign video information | SRLP (Sylvia Rivera Law Project), n.d.; Gehi and Arkles, 2007). For example, after the Advocate tweeted a link to an op-ed by the Executive Director of the Center for Transgender Equality titled, "Black, poor, young, trans is sadly often a recipe for violence: CeCe McDonald was punished for surviving," multiple members of the \#GirlsLikeUs network manually modified the tweet (MT) by adding \#FreeCeCe and \#GirlsLikeUs before sharing it. In this way members of the counterpublic insured that the narratives about violence against Black trans women that arose from more mainstream LGBTQ spaces were pulled into the intersectional discussions of their specific network.

Those tweeting \#GirlsLikeUs alongside \#FreeCeCe engaged in open criticism of narratives about McDonald's case arising from non-LGBTQ spaces, including critiques of the media's misgendering of McDonald through the use of incorrect pronouns and the sharing of petitions and other direct forms of advocacy related to the case. As Fischer (2016) has detailed in her ethnography of the CeCe Support Committee, a grassroots group of Minneapolis activists who took up advocating for McDonald during her imprisonment and trial, online activism in the case successfully motivated, shaped, and changed local and national coverage of the story.

Further, the co-occurrence of \#GirlsLikeUs and \#FreeCeCe hashtags was employed by members of the network to legitimize trans activism. For example, @ Arizona_Abby, a member of the \#GirlsLikeUs network who describes herself in her Twitter bio as, "Attorney, trans woman and general rabble-rouser on feminism, trans and LGB issues \#girlslikeus \#Trans 100," tweeted: 
Figure 5: Co-occurrence of \#GirlsLikeUs and \#FreeCece as activism

Here @ Arizona_Abby recalls the power of the AIDS Coalition to Unleash Power (ACT UP), one of the most revered, successful (and to some radical) LGBTQ activist groups of the twentiethcentury, to legitimize refusing to be silent on the cases of $\mathrm{CeCe}$ McDonald and Paige Clay, a Black transgender woman who was murdered in Chicago. Alongside the hashtagging of these two women's names and ACT UP, \#GirlsLikeUs appears in the tweet to signify McDonald and Clay's belonging in the counterpublic and to draw others' attention to their stories.

Notably, \#FreeCeCe has remained an important hashtag past the period of McDonald's incarceration. Laverne Cox has since produced the documentary Free Cece (2016), which documents McDonald's time in prison and subsequent release. The hashtag is used to promote the documentary and further activism on behalf of trans women still embroiled in the prison system.

\section{\#RedefiningRealness and \#TransisBeautiful}

In November of 2013, in concert with the launch of her book Redefining Realness: My Journey into Womanhood, Janet Mock began a social media campaign with the hashtag \#RedefiningRealness. The Tumblr instantiation of the hashtag invited people to post selfies that were overlayed with text that read, "I am [the person's name followed by a list of descriptors] I am \#RedefiningRealness." 


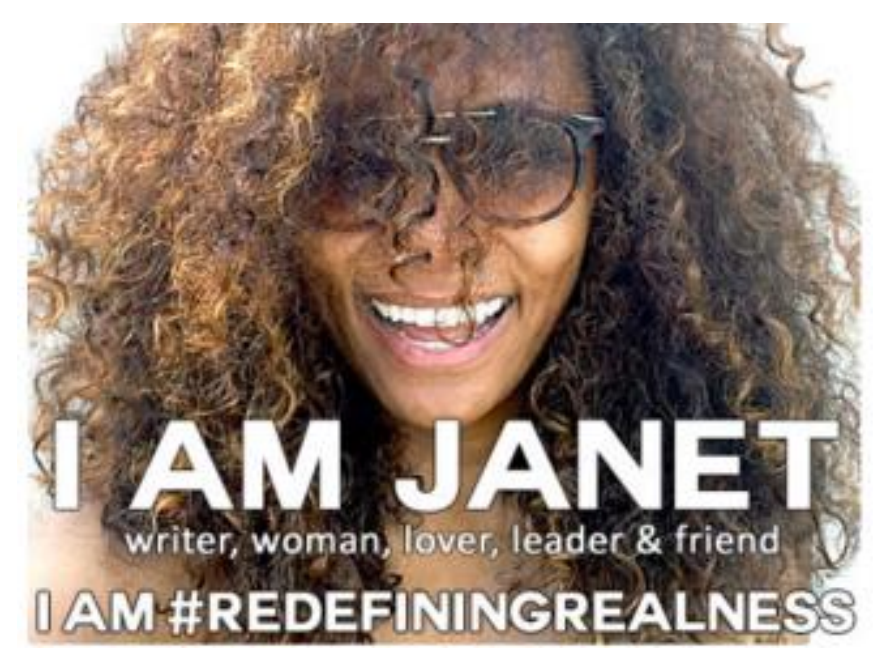

Figure 6: Janet Mock Tumblr post

Mock began the social media initiative with a selfie that read, "I AM JANET writer, woman, lover, friend I am \#REDEFININGREALNESS," (I AM \#RedefiningRealness, n.d.). A notably diverse group of users submitted to the Tumblr site for nearly ten months with over 120 unique contributions. The hashtag encouraged users to say more about themselves than their gender or sexuality, centering their humanity and various social and relational roles. Much like the community-building and celebrating functions of \#GirlsLikeUs, contributors discussed their jobs, their favorite qualities about themselves, and their hopes for the future. The use of the hashtag \#RedefiningRealness quickly moved to Twitter where it is used by trans and queer users to describe ways in which they are redefining gender, fans of Mock's posting selfies with the book, and in Mock's own tweets logging her nationwide travels to promote the book and related projects.

In a similar spirit, Laverne Cox started the \#TransIsBeautiful hashtag in 2015 after discussing her past shame at being physically identifiable as a trans woman. She observed: It took me years to fully internalize that someone can look at me and tell that I am transgendered and that is not only okay, but beautiful, because trans is beautiful. All the things that make me uniquely and beautifully trans - my big hands, my big feet, my wide shoulders, my deep voice- 
all of these things are beautiful. I'm not beautiful despite these things, I'm beautiful because of them. (Chernikoff, 2015)

As in the case of Mock and \#RedefiningRealness, Cox uses a hashtag to advocate for the humanity and value of trans women by insisting that mainstream and cis-normative definitions of womanhood and beauty be confounded and expanded. Of course, part of what Mock and Cox are doing with \#RedefiningRealness and \#TransIsBeautiful is also strategic self-promotion; they are leveraging their social media followings to promote their personal brands and products (Schawbel, 2009). Janet Mock was interviewed about \#RedefiningRealness on almost every major network and cable channel after the hashtag became popular in the network, and \#TransIsBeautiful has been featured in mainstream fashion magazines like Elle and pop culture magazines like People. As successful trans women of color, this self-promotion also does important community building and cultural interventionist work. As they share their successes, Mock and Cox encourage their followers to share discourses about learning to love themselves and finding appreciation for and community with other trans women. The particular affordances of Twitter allow these exchanges to be simultaneously intimate and public, promoting brands and also supporting trans women in need of social and other forms of support (Marwick and Boyd, 2011).

Many members of the \#GirlsLikeUs network tweeted links sharing their own donations, and encouraging their followers to donate to \#TransBookDrive, an effort begun by Mock to insure that low-income and incarcerated members of the trans community could access Redefining Realness and any other books they requested. This effort and the tweets it inspired with the co-occurring hashtags \#GirlsLikeUs and \#RedefiningRealness are indicative of the acknowledgement within the trans feminist counterpublic that many of its members lack support 
and resources for even the simplest of self-affirming resources, like books that center their stories.

The depiction of Laverne Cox, a Black trans women known primarily for playing a prisoner as Lady Liberty, one of the most beloved American political and cultural symbols, on the cover of Entertainment Weekly's first ever "LGBT Issue" speaks to the interventionist power of \#GirlsLikeUs. Black trans women and their political frameworks have not only become the face of trans issues, but of LGBT issues, and the nation as a whole, a representation few could have imagined in 2012 when \#GirlsLikeUs began. ${ }^{7}$

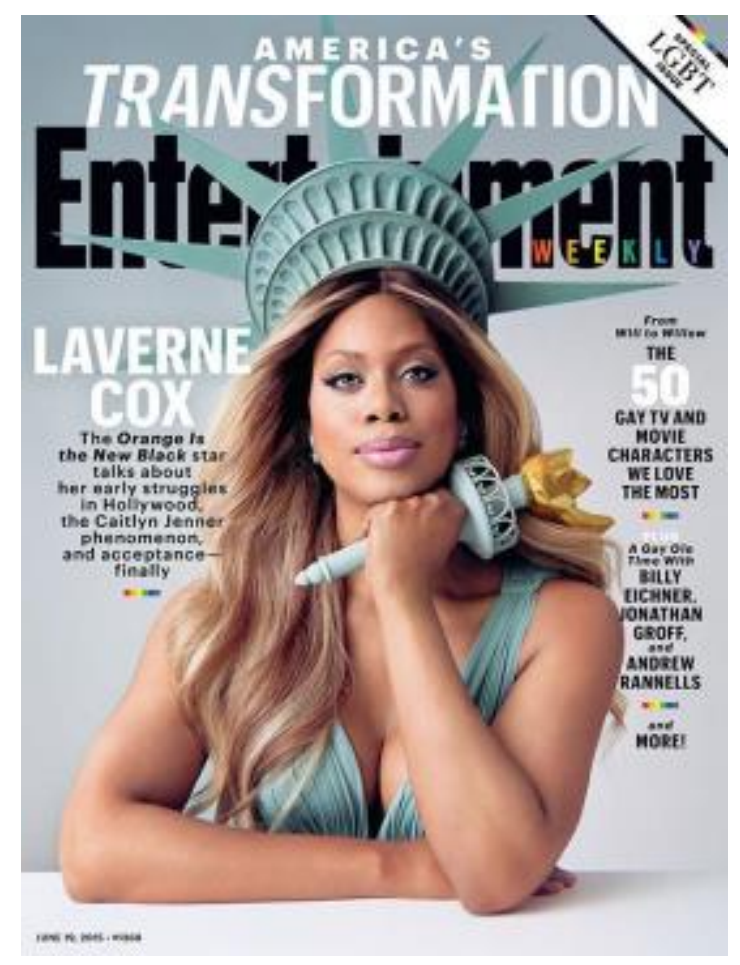

Figure 7: Laverne Cox as Lady Liberty

Thus, vis-à-vis their growing visibility and self-promotion in the \#GirlsLikeUs network, Mock and Cox encourage and extend the larger modes of social support offered by the trans feminist counterpublic and do important cultural work in regard to mainstream representation. 
While this support often includes celebrating the style choices, successes, and physical beauty of trans women, it also frequently includes members of \#GirlsLikeUs using co-occurrence to lift up, support, and call for help for members of the network who might be struggling with mainstream messages and conditions related to the oppression of trans identity.

\section{Conclusion: The Power of \#GirlsLikeUs}

Ultimately, the discursive work done by members of the \#GirlsLikeUs counterpublic is a striking embodiment of the feminist principle that "the personal is political." By using the hashtag to mark the mundane, the popular, and the activist within the network, tweets work to both center trans politics and normalize trans lives. It is clear from the discursive and network data that \#GirlsLikeUs prioritized in-group solidarity from day one, even as its members began to engage the broader public. In-group messages spread beyond their initial community and were intentionally and widely shared in moments of advocacy, like that on behalf of CeCe McDonald, and more generally because of the public nature of Twitter. The technological affordances of Twitter simultaneously served as a conduit through which trans women connected and supported one another and a channel for broadcasting their messages to broad audiences.

Our findings show that in building a community that transcends distances, the contributions of members of the \#GirlsLikeUs network perform important cultural and interpersonal work by constructing and extending a trans feminist counterpublic that works toward self-sustaining identity creation, and in doing so, supports the health and social inclusion of network members. At the same time this network worked, over time, toward elevating the stories and needs of trans women into the mainstream - as previously noted, mainstream media sources that responded to and echoed the language of the network appeared in the Twitter data collected here and many members of the network tweeted to and about these sources. For 
example, a Rolling Stone story on CeCe McDonald that was published after her release from prison was shared in the network with the co-occurring hashtags \#FreeCeCe and \#GirlsLikeUs. Further, the mainstream visibility and promotional campaigns of some members of the counterpublic, Mock and Cox in particular, further facilitated the visible interrogation of mainstream narratives about gender identity and feminist and queer politics while supporting ingroup needs.

Importantly, this research also reveals that the trans feminist network that sustains and is sustained by \#GirlsLikeUs is one that centers discourses from the most multiply marginalized trans voices. Influencers in this network are not only trans, but women, Black, and in some cases quite open about their experiences with poverty, sex work, and the legal system. As a collective, the network facilitates and encourages ordinary trans women, with a variety of intersectional identities, to speak up and out about their experiences in a networked counterpublic that has become much bigger than they could have predicted.

As part of a larger cultural shift that centers sex, gender, and civil rights, and which has been led primarily by trans women of color, the network that emerged around the hashtag \#GirlsLikeUs plays an indispensable role in constructing an intersectional networked trans community. \#GirlsLikeUs simultaneously works to shift representations of trans people that arise from the public sphere. Ultimately, though the network was partially centered around the rising visibility of some members of its community, its story is one of collective support and advocacy. Through their conversations and retweets, thousands of members of the \#GirlsLikeUs network lifted up the voices of members that represented their needs and experiences, and ensured that these transcended their subaltern origins.

\section{References}


Asen R and Brouwer DC (2001) Counterpublics and the State. SUNY Press.

Bailey M (2015) \#transform(ing)DH Writing and Research: An Autoethnography of Digital Humanities and Feminist Ethics. 9(2). Available from: http://www.digitalhumanities.org/dhq/vol/9/2/000209/000209.html (accessed 14 December 2015).

Bailey M (2016) Redefining Representation: Black Trans and Queer Women's Digital Media Production. Screen Bodies 1(1). Available from: http://berghahnjournals.com/view/journals/screenbodies/1/1/screen010105.xml (accessed 18 October 2016).

Barabási A-L and Albert R (1999) Emergence of scaling in random networks. science 286(5439): 509512.

Barnett JT (2015) Fleshy Metamorphosis: Temporal Pedagogies of Transsexual Counterpublics. In: Spencer LG and Capuzza JC (eds), Transgender communication studies: Histories, trends, and trajectories, pp. 155-172.

Benkler Y (2006) The wealth of networks: How social production transforms markets and freedom. Yale University Press. Available from:

https://books.google.com/books?hl=en\&lr=\&id=Q08oChJj8HQC\&oi=fnd\&pg=PT2\&dq=The+w ealth+of+networks:+How+social+production+transforms+markets+and+freedom\&ots=qhkYVpXFL\&sig=w0t8CtG1fFbOdPi52iUTA31vyw8 (accessed 18 October 2016).

Booth J (2015) Exploring Intersectional Relationships Between Experiences of Racial and Gbt (Gay, Bisexual, Transgender) External and Internalized Discrimination. In: Society for Social Work and Research 19th Annual Conference: The Social and Behavioral Importance of Increased Longevity, Sswr.

Capuzza JC and Spencer LG (2015) Transgender Communication Studies: Histories, Trends, and Trajectories. Lexington Books.

Cavalcante A (2016) 'I Did It All Online:' Transgender identity and the management of everyday life. Critical Studies in Media Communication 33(1): 109-122.

Chernikoff L (2015) Laverne Cox's Explanation of Why \#TransIsBeautiful Will Make You Cheer and Cry. ELLE. Available from: http://www.elle.com/culture/celebrities/news/a30388/laverne-coxtrans-is-beautiful/ (accessed 20 July 2016).

Cox L (2013) Check out the video of my \#nerdland debut today. We talk about @OITNB and prison policy http://video.msnbc.msn.com/mhp/52670030\#52670030 ...@MHPshow \#girlslikeus. microblog. Available from: https://twitter.com/search?q=\%23BecauseofCeCe\&src=hash (accessed 22 October 2016).

Crenshaw K (1991) Mapping the margins: Intersectionality, identity politics, and violence against women of color. Stanford Law Review 43(6): 1241-1299.

End Solitary campaign video information | SRLP (Sylvia Rivera Law Project) (n.d.). Available from: http://srlp.org/endsolitary/videoinformation/ (accessed 3 August 2015). 
Ennis D (2015) Booing Jennicet Was Wrong, But Was What She Did Worse? Available from: http://www.advocate.com/commentary/2015/07/01/booing-jennicet-was-wrong-was-what-shedid-worse (accessed 20 July 2016).

Fischer M (2016) \#Free_CeCe: the material convergence of social media activism. Feminist Media Studies 16(5): 755-771.

Florini S (2014) Recontextualizing the Racial Present: Intertextuality and the Politics of Online Remembering. Critical Studies in Media Communication 31(4): 314-326.

Gamson J (1998) Freaks Talk Back: Tabloid Talk Shows and Sexual Nonconformity. 2nd ed. edition. Chicago: University of Chicago Press.

Gehi PS and Arkles G (2007) Unraveling injustice: Race and class impact of medicaid exclusions of transition-related health care for transgender people. Sexuality Research \& Social Policy 4(4): 7.

Heinz M (2015) Interpersonal Communication: Trans Interpersonal Support Needs. Available from: https://www.researchgate.net/publication/274005950_Interpersonal_Communication_Trans_Inter personal_Support_Needs (accessed 16 August 2016).

Hill ML (2012) Why Aren't We Fighting for CeCe McDonald? EBONY. Available from: http://www.ebony.com/news-views/why-arent-we-fighting-for-cece-mcdonald (accessed 25 October 2016).

Hundley HL and Rodriguez JS (2009) Transactivism and postmodernity: An agonistic analysis of transliterature. Communication Quarterly 57(1): 35-50.

I AM \#RedefiningRealness (n.d.). Available from: http://redefiningrealness.tumblr.com/?og=1 (accessed 1 February 2014).

Jackson SJ (2014) Black Celebrity, Racial Politics, and the Press: Framing Dissent. 1 edition. New York, NY: Routledge.

Jackson SJ and Foucault Welles B (2015) Hijacking\# myNYPD: Social media dissent and networked counterpublics. Journal of Communication 65(6): 932-952.

Jackson SJ and Foucault Welles B (2016) \#Ferguson is everywhere: initiators in emerging counterpublic networks. Information, Communication \& Society 19(3): 397-418.

Johnson JR (2013) Cisgender privilege, intersectionality, and the criminalization of CeCe McDonald: Why intercultural communication needs transgender studies. Journal of International and Intercultural Communication 6(2): 135-144.

Lee C and Kwan PKY (2014) The Trans Panic Defense: Heteronormativity, and the Murder of Transgender Women. The Trans Panic Defense: Heteronormativity, and the Murder of Transgender Women. Available from: http://papers.ssrn.com/sol3/papers.cfm?abstract_id=2430390 (accessed 2 April 2015).

Loader BD and Mercea D (2011) Networking Democracy? Information, Communication \& Society 14(6): 757-769. 
Martohardjono Z and Young R (2016) Toward Transfeminism: Moving Beyond Inclusion| Nonprofit Quarterly. Non Profit News For Nonprofit Organizations | Nonprofit Quarterly. Available from: https://nonprofitquarterly.org/2016/03/02/toward-transfeminism-moving-beyond-inclusion/ (accessed 25 October 2016).

Marwick AE and Boyd D (2011) I tweet honestly, I tweet passionately: Twitter users, context collapse, and the imagined audience. New media \& society 13(1): 114-133.

Mendelson S (2015) Why Roland Emmerich's ‘Stonewall' Was A Box Office Disaster. Forbes. Available from: http://www.forbes.com/sites/scottmendelson/2015/09/29/why-roland-emmerichs-stonewallwas-a-box-office-disaster/ (accessed 12 March 2017).

Michaels S (2015) It's incredibly scary to be a transgender woman of color right now. Mother Jones. Available from: http://www.motherjones.com/politics/2015/06/transgender-womendisproportionately-targeted-violent-hate-crimes (accessed 22 June 2016).

Mock J (2012) Why I Started \#GirlsLikeUs Twitter Hashtag For Trans Women | Janet Mock. Available from: http://janetmock.com/2012/05/28/twitter-girlslikeus-campaign-for-trans-women/ (accessed 11 August 2014).

Mock J (2013) Dry those tears, babydoll. You are a bright, shining star, @ xocassidylynn! http://laist.com/2013/09/21/transgender_teen_voted_homecoming_q.php ... \#girlslikeus. microblog. Available from: https://twitter.com/search?q=\%23BecauseofCeCe\&src=hash (accessed 22 October 2016).

Mock J (2014a) \#BecauseofCeCe I was inspired to begin using the phrasing \#GirlsLikeUs which led to a social media visibility movement. \#CeCeIsFree. microblog. Available from: https://twitter.com/search?q=\%23BecauseofCeCe\&src=hash (accessed 22 October 2016).

Mock J (2014b)@GeenaRocero gorgeous + glowing! Can't wait to pick up my copy! \#genderproud \#girlslikeus. microblog. Available from: https://twitter.com/search?q=\%23BecauseofCeCe\&src=hash (accessed 22 October 2016).

Papacharissi Z and de Fatima Oliveira M (2012) Affective news and networked publics: The rhythms of news storytelling on\# Egypt. Journal of Communication 62(2): 266-282.

Rawson KJ (2014) Transgender Worldmaking in Cyberspace: Historical Activism on the Internet. QED: A Journal in GLBTQ Worldmaking 1(2): 38-60.

Romero DM and Kleinberg J (2010) The Directed Closure Process in Hybrid Social-Information Networks, with an Analysis of Link Formation on Twitter. arXiv:1003.2469 [physics, stat]. Available from: http://arxiv.org/abs/1003.2469 (accessed 18 October 2016).

Ross A (2013) I'm regaining at least 20 hours of my life back a week moving to Chicago! More time for my spirit, tennis, yoga \& friends \#GirlsLikeUs. microblog. Available from: https://twitter.com/ (accessed 25 October 2016).

Ross A (2014) Just interviewed by @ Forbes Magazine on @TransTechSocial \& my journey as a black trans entrepreneur! Thanks @ClairJoyFarley! \#GirlsLikeUs. microblog. Available from: https://twitter.com/search?q=\%23BecauseofCeCe\&src=hash (accessed 22 October 2016). 
San Francisco Trans March (n.d.) Mission. Available from: http://www.transmarch.org/mission/ (accessed 22 October 2016).

Schawbel D (2009) Me 2.0: Build a Powerful Brand to Achieve Career Success. Original edition. New York: Kaplan Publishing.

Schilt K and Westbrook L (2009) Doing Gender, Doing Heteronormativity: 'Gender Normals,' Transgender People, and the Social Maintenance of Heterosexuality. Peer Reviewed Articles. Available from: http://scholarworks.gvsu.edu/soc_articles/7.

Serano J (2007) Whipping Girl: A Transsexual Woman on Sexism and the Scapegoating of Femininity. annotated edition edition. Emeryville, CA: Seal Press.

Shorey E (2015) Backlash Against 'Stonewall' Prompts Donations To 'Happy Birthday, Marsha!' LOGO News. Available from: http://www.newnownext.com/backlash-against-stonewall-promptsdonations-to-happy-birthday-marsha/08/2015/ (accessed 16 August 2016).

Smith MA, Rainie L, Shneiderman B, et al. (2014) Mapping Twitter Topic Networks: From Polarized Crowds to Community Clusters. Pew Research Center: Internet, Science \& Tech. Available from: http://www.pewinternet.org/2014/02/20/mapping-twitter-topic-networks-from-polarized-crowdsto-community-clusters/ (accessed 16 August 2016).

Southern Poverty Law Center (2015) Murders of Trans Women This Year Hits New High. Southern Poverty Law Center. Available from: https://www.splcenter.org/fighting-hate/intelligencereport/2015/murders-trans-women-year-hits-new-high (accessed 22 June 2016).

Squires CR (2002) Rethinking the Black Public Sphere: An Alternative Vocabulary for Multiple Public Spheres. Communication Theory 12(4): 446-468.

Statista (2015) Most retweeted posts 2015 | Statistic. Available from: http://www.statista.com/statistics/493795/twitter-most-retweeted-posts/ (accessed 16 August 2016).

Vitulli E (2010) A Defining Moment in Civil Rights History? The Employment Non-Discrimination Act, Trans-Inclusion, and Homonormativity. Sexuality Research and Social Policy 7(3): 155-167.

Warner M (2002) Publics and Counterpublics. Public Culture 14(1): 49-90.

\footnotetext{
${ }^{1}$ As scholars, we are committed to ethical research practices. As such we consulted with Janet Mock before beginning this research project and received her consent for our continued analysis.

2 \#girlslikeus as a hashtag predates Janet Mock's deployment. It was initially a little used hashtag to promote the film Girls Like Us, a short lived now squelched Joni Mitchell biopic set to star Taylor Swift. The studio attempted to make the hashtag popular by repeatedly tweeting the tag and getting Swift fans to reblog it. However, no one created original tweets with the hashtag and when the project was shelved, there was little trace of the tag when Mock generated it herself. This demonstrates the ephemeral nature of hashtag use if the particular initiating use of a tag does not resonate widely.
} 
${ }^{3}$ In fact, Sylvia Rivera, founding member of some of the first gay and trans liberation organizations in America was once similarly booed and chastised for speaking to the concerns of trans women of color at a pride rally in 1973, only a few short years after her pivotal role in starting the LGBTQ movement.

4 "Is 'Transface' a Problem in Hollywood?," February 25, 2015, http://www.advocate.com/artsentertainment/2015/02/25/transface-problem-hollywood. Notably, during his Emmy acceptance speech for his role in Transparent in 2016, Jeffrey Tambour argued passionately that he should be the last cisgender man to play a transwoman in television and film.

${ }^{5}$ Like other groups, class status and race impact the lives of different trans women and shape their political perspectives differently. For example, Caitlin Jenner's conservatism has prompted confusion, including during a 2015 interview with Ellen Degeneres after Jenner mentioned her own reticence around the issue of gay marriage, prompting Degeneres to say she expected Jenner to have a more left leaning political stance upon transitioning. Not only did Degeneres conflate Caitlin's transition with a liberal political perspective, but also with her views on sexuality.

${ }^{6}$ Our Commitment to ethical research practices includes not revealing the names of Twitter users with vulnerable identities who have not already come under some form of public attention and/or who have not provided their consent to be named in this research. Please see Bailey (2015), "\#transform(ing)DH Writing and Research: An Autoethnography of Digital Humanities and Feminist Ethics," where this issue is discussed further.

${ }^{7}$ Future research should more expansively detail the relationship between particularly subaltern counterpublics and notable mainstream shifts in identity representation and politics. 